\title{
Pityriasis Rosea Induced by COVID-19 Vaccination
}

\author{
Elina Khattab ${ }^{1}$, Eirini Christaki ${ }^{2}$, Constantinos Pitsios ${ }^{1}$ \\ ${ }^{1}$ Medical School, University of Cyprus, Nicosia, Cyprus \\ ${ }^{2}$ Department of Internal Medicine, Faculty of Medicine, School of Health Sciences, University of loannina, loannina, Greece
}

Received: 03/01/2022

Accepted: $12 / 01 / 2022$

Published: 03/02/2022

How to cite this article: Khattab E, Christaki E, Pitsios C. Pityriasis rosea induced by COVID-19 vaccination. EJCRIM 2022;9: doi:10.12890/2022_003164.

Conflicts of Interests: The authors declare there are no competing interests.

This article is licensed under a Commons Attribution Non-Commercial 4.0 License

\section{ABSTRACT}

It is increasingly recognized that SARS-CoV-2 infection and COVID-19 vaccines have been associated with skin disorders, including pityriasis rosea. It has been reported that pityriasis rosea has been triggered by several vaccines, as a rare side-effect. We present two cases of COVID-19 vaccine-induced pityriasis rosea. Skin lesions appeared in a 49-year-old female 8 days after the first dose of the BNT162b2 mRNA vaccine and in a 53-year-old male 7 days after the second dose of the same vaccine. The exanthem was self-limited in both patients over a period of a month.

\section{LEARNING POINTS}

- Physicians should be aware that pityriasis rosea is a rare side-effect of COVID-19 vaccination.

- Pityriasis rosea is self-limiting and no medical treatment is usually required.

\section{KEYWORDS}

COVID-19, SARS-CoV-2, vaccination, pityriasis rosea

\section{INTRODUCTION}

The efficacy and safety of COVID-19 vaccines are currently a scientific priority. A large body of evidence has emerged in late 2021 to show that SARS-CoV-2 infection and COVID-19 vaccines are associated with skin disorders, including pityriasis rosea (PR) and pityriasis rosealike eruptions (PR-LE) ${ }^{[1-6]}$. Vaccination-induced cases of PR by other vaccines have also been reported in the past[7].

$\mathrm{PR}$ is a self-limiting benign skin disorder commonly seen in clinical practice ${ }^{[8,9]}$. It affects approximately $0.5-2 \%$ of the general population and appears to be more common in young adults than in the elderly and children ${ }^{[8]}$. The disease starts with the sudden appearance of a single erythematous patch known as the 'herald patch', which is followed by secondary scaly lesions with the characteristic 'Christmas tree' distribution on the cleavage lines ${ }^{[8,9]}$.

The precise etiopathogenesis of PR remains largely unknown and its etiology is hypothesized to be multifactorial, including both infective and non-infective factors, such as autoimmunity and atopy ${ }^{[1,8]}$. The activation of immunological mechanisms triggers PR in both cases: infection and vaccination. Here we report two cases of PR which developed following the administration of BNT162b2 (BioNTech, Mainz, Germany; Pfizer, New York City, NY, USA) vaccine.

\section{CASE DESCRIPTIONS}

A 49-year-old Caucasian female presented for evaluation of a macular rash initially distributed on her trunk, which a day later extended to her extremities with the onset of many itchy scaly lesions (Fig. 1). The patient had noticed an oval scaly herald lesion on her abdomen, $2.5 \mathrm{~cm}$ in diameter, appearing 2 days before the rash. Eight days prior to the appearance of the herald patch she had received the first dose of the BNT162b2 vaccine. The patient had no known medical issues or allergies and was not on medication. 


\section{European Journal}

of Case Reports in

Internal Medicine

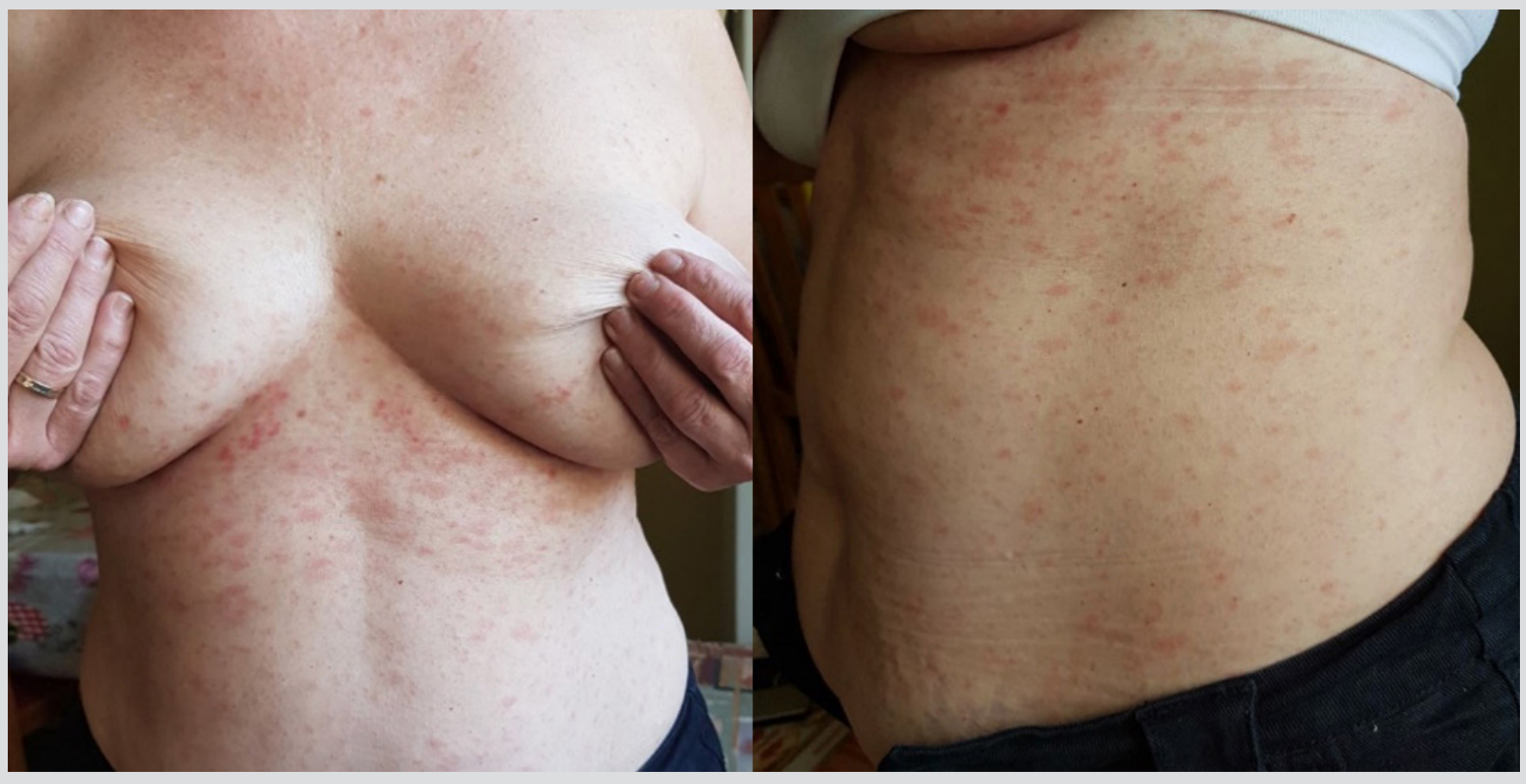

Figure 1. Herald patch in the right hypochondrium followed by typical oval-shaped secondary plaques

On clinical examination, she was afebrile and her skin lesions were compatible with a diagnosis of PR. She was treated with oral antihistamines and topical betamethasone $0.1 \%$, with clinical improvement. Her rash gradually improved and at follow-up, 4 weeks later, it had resolved completely. She received a delayed second dose of the vaccine, 45 days after the first dose, when no lesions remained. On communication 2 weeks later, she reported no vaccine-related side-effects.

A 53-year-old Caucasian male presented with an itchy macular rash on his upper trunk and abdomen. A herald annular plaque was observed on his upper abdomen and a diagnosis of PR was made. The lesions had appeared 7 days after the second dose of the BNT162b2 vaccine. Within 48 hours, it had extended to his extremities (Fig. 2). His medical history included transient ischemic attacks, coronary artery disease, and dyslipidemia, for which he was taking medication. The itching was self-limiting and therefore the patient did not receive any antihistamines. His rash resolved completely over a period of 4 weeks.

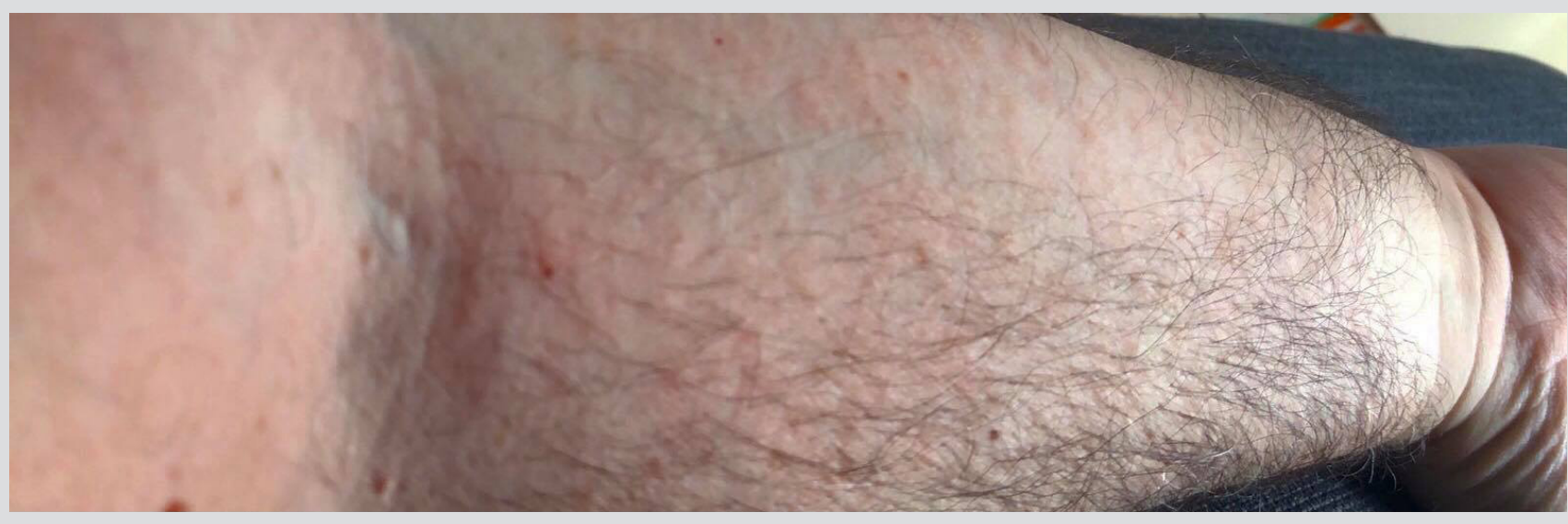

Figure 2. Pityriasis rosea lesions on the upper extremities 


\section{DISCUSSION}

PR is a self-limiting papulosquamous skin disorder where the reactivation of latent human herpes virus (HHV 6/7) infection is considered the most likely etiological agent ${ }^{[9]}$. SARS-CoV-2 infection as well as immunization with COVID-19 vaccines have been associated with cases of cutaneous adverse events, including PR and PR-LEE ${ }^{[2,3,10]}$. It is unclear if the pathophysiology leading to PR is the same. In the case of infection, a cytokine storm is observed, while in the case of immunization the released cytokines are in the frame of immune stimulation.

The viral pathophysiology of SARS-CoV-2 is characterized by the simultaneous occurrence of cytokine release and immunosuppression. This suppressed and dysregulated immune state may trigger the reactivation of latent viral infections, such as HHV 6 and HHV $7^{[10]}$. Factors supporting the viral reactivation theory include positive tissue immunohistochemistry, the detection of in situ viral mRNA, and IgG antibodies against $\mathrm{HHV} 6 / 7^{[1]}$. Moreover, the detection of the SARS-CoV-2 virus spike protein on endothelial cells and lymphocytes indicates a direct correlation between COVID-19 infection and PR ${ }^{[3]}$. Increasing evidence connecting SARS-CoV-2 to PR should raise questions about PR etiology and encourage an evaluation of the association between PR and seasonal coronaviruses.

The exact pathogenic mechanism leading to $P R$ after vaccination remains unclear ${ }^{[7]}$. The main hypothesis is that COVID-19 vaccines may induce PR by reactivation of latent viral infections, mainly HHV 6/7 $7^{[1,7]}$. In some cases, antibodies against HHV 6/7 and viral DNA in plasma and neutrophils were detected. These findings support an immune-induced reactivation of latent viral infections ${ }^{[1]}$. An immunomodulatory effect of vaccination has been proposed, characterized by a vaccine-induced distraction of the cell-mediated control on latent infections. Another possible etiology is that vaccination-induced PR may occur due to a specific T-cell-mediated immune response, triggered by molecular mimicry. Physicians should be aware that PR may appear after any dose (initial or booster) of the mRNA vaccine.

Several cases of PR/PR-LE, limited to 29 according to a review, have been reported after vaccinations for smallpox, poliomyelitis, influenza, papillomaviruses, diphtheria, tuberculosis, hepatitis B, pneumococcus, and yellow fever ${ }^{[7]}$. To our knowledge, there are at least six cases of PR reported after mRNA-based COVID-19 vaccines - Pfizer/BioNTech (BNT162b2) and Moderna (mRNA-1273) (Moderna, Cambridge, MA, USA) - and one case after CoronaVac vaccine (Sinovac Life Sciences, Beijing, China) ${ }^{[1,4-6,11]}$. The number of PR cases due to COVID-19 vaccines is disproportionally high compared with other vaccines; however, the explanation may be very simple. The global scientific community has tried to extensively study all the parameters of COVID-19 and medical journals have strongly accelerated their publication processes. We believe that the reports are an epiphenomenon of the increased awareness of the vaccine side-effects.

Given the importance and urgency of widespread vaccination due to the pandemic, recognizing and managing COVID-19 vaccine-induced complications is beyond doubt. Recent evidence, arising primarily from case reports, suggest that there is a correlation between SARSCoV-2 infection, COVID-19 vaccines, and exanthematous disease, including PR. However, the etiopathogenic mechanisms remain largely unknown and further immunological studies are needed in order to clarify the correlation between vaccination and PR.

\section{REFERENCES}

1. Busto-Leis JM, Servera-Negre G, Mayor-Ibarguren A, Sendagorta-Cudós E, Feito-Rodríguez M, Nuño-González A, et al. Pityriasis rosea, COVID-19 and vaccination: new keys to understand an old acquaintance. J Eur Acad Dermatol Venereol 2021;35(8):e489-e491.

2. Johansen M, Chisolm SS, Aspey LD, Brahmbhatt M. Pityriasis rosea in otherwise asymptomatic confirmed COVID-19-positive patients: a report of 2 cases. JAAD Case Rep 2021;7:93-94.

3. Welsh E, Cardenas-de la Garza JA, Cuellar-Barboza A, Franco-Marquez R, Arvizu-Rivera RI. SARS-CoV-2 spike protein positivity in pityriasis rosea-like and urticaria-like rashes of COVID-19. Br J Dermatol 2021;184(6):1194-1195.

4. Carballido Vázquez AM, Morgado B. Pityriasis rosea-like eruption after Pfizer-BioNTech COVID-19 vaccination. Br J Dermatol 2021:185(2):e34

5. Cyrenne BM, Al-Mohammedi F, DeKoven JG, Alhusayen R. Pityriasis rosea-like eruptions following vaccination with BNT162b2 mRNA COVID-19 vaccine. J Eur Acad Dermatol Venereol 2021;35(9):e546-e548.

6. Akdaş E, Illter N, Öğüt B, Erdem Ö. Pityriasis rosea following CoronaVac COVID-19 vaccination: a case report. J Eur Acad Dermatol Venereol 2021;35(8):e491-e493.

7. Drago F, Ciccarese G, Javor S, Parodi A. Vaccine-induced pityriasis rosea and pityriasis rosea-like eruptions: a review of the literature. J Eur Acad Dermatol Venereol 2016;30(3):544-545.

8. Mahajan K, Relhan V, Relhan AK, Garg VK. Pityriasis rosea: an update on etiopathogenesis and management of difficult aspects. Indian J Dermatol 2016;61(4):375-384.

9. Goldstein AO, Goldstein BG. Pityriasis rosea. In: R. Dellavalle, ML Levy, AO Ofori (Eds), UpToDate. Assessed: January 22 nd, 2022.

10. Sugawara-Mikami M, Ishii N, Yamazaki M, Kambara T, Sasaki H, Tachikawa N et al. Skin manifestations of suspected COVID-19: complications of the disease or reactivation of latent viral infections? JAAD Case Rep 2021;12:15-17.

11. Papakostas D, Stavropoulos PG, Papafragkaki D, Grigoraki E, Avgerinou G, Antoniou C. An atypical case of pityriasis rosea gigantea after influenza vaccination. Case Rep Dermatol 2014;6:119-123. 